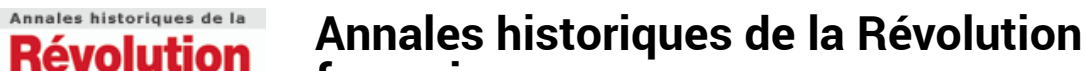

française française

324 | avril-juin 2001

Louis Charles Antoine Desaix. Officier du roi, Général de la République

\section{Le sultan dévoilé}

Desaix en Égypte d'après ses notes de campagne

Philippe Bourdin

\section{(2) OpenEdition \\ Journals}

\section{Édition électronique}

URL : https://journals.openedition.org/ahrf/365

DOI : $10.4000 /$ ahrf.365

ISSN : 1952-403X

Éditeur :

Armand Colin, Société des études robespierristes

Édition imprimée

Date de publication : 1 juin 2001

Pagination : 47-62

ISSN : 0003-4436

Référence électronique

Philippe Bourdin, «Le sultan dévoilé », Annales historiques de la Révolution française [En ligne], 324 |

avril-juin 2001, mis en ligne le 22 mai 2006, consulté le 24 avril 2022. URL : http://

journals.openedition.org/ahrf/365; DOI : https://doi.org/10.4000/ahrf.365

Ce document a été généré automatiquement le 24 avril 2022.

Tous droits réservés 


\title{
Le sultan dévoilé
}

Desaix en Égypte d'après ses notes de campagne

\author{
Philippe Bourdin
}

1 Le 26 mai 1798, Louis Charles Antoine Desaix quitte Cività-Vecchia et, avec soixante navires, rejoint Bonaparte à Malte pour tenter l'aventure égyptienne. Son nom sera désormais associé à la plupart des hauts faits militaires au cours desquels son courage sera indéniable, à l'image de beaucoup d'autres soldats connus et inconnus. Il participera tout autant aux représailles et aux exactions de la campagne, auxquelles s'appliquent les camps adverses. Car sa guerre n'est pas belle, d'Alexandrie le 1er juillet à la bataille des Pyramides le 21, du Caire le lendemain à l'ininterrompue et harassante poursuite de Mûrad Bey en haute Égypte, occasion entre septembre 1798 et mars 1799 de victoires éclatantes mais toujours insuffisantes, comme celles de Sediman, le 16 octobre, ou de Samhûd, le 21 janvier. Le temps de s'emparer personnellement de quelques trophées précautionneusement ramenés en France : la masse d'armes, le sabre et l'étendard de Mûrad-Bey par exemple ${ }^{1}$... Jusqu'à l'ultime échec, dont Desaix, plénipotentiaire sous Kléber, devra négocier malgré lui les premières conséquences avec les Turcs et les Anglais, le 18 Brumaire passé : ce sera la convention d'al-'Arîsh qui, le 4 pluviôse an VIII (24 janvier 1800), organisera l'évacuation des armées françaises des rives du Nil, de la mer Rouge et de la Méditerranée. Demeurent, avant l'inscription dans la postérité largement organisée par Bonaparte, les témoignages de ce dernier et de quelques autres compagnons de route, les notes surtout, prises sur le vif des opérations, d'une écriture jetée à la hâte par Desaix lui-même lors des rares haltes et des bivouacs inconfortables: soixante-dix huit pages Sur les diverses tribus arabes de l'Égypte, leur origine, leur étendue, leurs alliances et leurs guerres ; vingt-deux autres sur la Position de l'Armée française en Égypte. Moyens de conserver cette conquête ${ }^{2}$. Quelle image renvoientelles du militaire éclairé passé par l'école royale d'Effiat, du noble gagné à la République au contraire de ses frères émigrés?

2 Les journaux et les mémoires des vétérans de la campagne d'Égypte ne manquent pas ; rares sont ceux, sinon sans doute celui du général Belliard, à tenter des portraits sensibles de Desaix; il reste pour beaucoup le chef que l'on respecte et que l'on suit, sans autre commentaire - le Journal de l'expédition d'Égypte du général Jean-Pierre 
Doguereau illustre cette sobriété ${ }^{3}$. Aussi les plus belles pages restent celles que consacrera Napoléon à un subordonné avec lequel il avait entretenu des relations aussi amicales qu'orageuses et passionnées. "Personne n'était plus propre à diriger une pareille opération que Desaix; personne ne le désirait avec plus d'ardeur. Jeune, la guerre était sa passion ; insatiable de gloire, il connaissait toute celle qui était attachée à la conquête de ce berceau des arts et des sciences. Au seul nom de Thèbes, de Coptos, de Philae, son cœur palpitait d'impatience ", confessera-t-il dans ses Campagnes d'Égypte et de Syrie. Reviennent alors en mémoire les cadeaux et les lettres d'affection envoyés au divisionnaire qui conquiert la haute Égypte. Effacées au contraire les dissensions stratégiques d'août 1799 lorsque Bonaparte rappelait vertement les limites de ses prérogatives à son obligé désobéissant ${ }^{4}$ - ce dernier n'en démordait pas: "Je ne m'excuserai pas ; vous n'êtes pas disposé à m'entendre ; je ne crains pas les torts et sais les avouer. J'ai fait cependant tout ce qui était le meilleur; toutes vos dispositions ont été exécutées à la lettre, sauf les retards, que les circonstances et la fortune, plus fortes que tout le monde, ont voulus $»^{5}$. Passés par pertes et profits les reproches justifiés par un impôt levé par l'Auvergnat sur les provinces occupées au bénéfice exclusif de sa division. Oubliée enfin la longue entrevue des retrouvailles italiennes du 11 juin 1800 à Montebello, où Desaix, depuis trop longtemps attendu en Europe, est sommé de s'expliquer sur son allégeance à Kléber et son rôle dans la capitulation :

«Dans la journée du 11, Desaix, qui revenait d'Égypte et qui avait fait la quarantaine à Toulon, arriva au quartier général de Montebello avec ses aides de camp Rapp et Savary. La nuit entière se passa en longues conférences entre le Premier consul et Desaix sur tout ce qui s'était passé en Égypte depuis que le Premier consul en était parti, sur les détails de la campagne de la haute Égypte, sur les négociations d'ElA'rych et la composition de la grande armée turque du grand vizir, enfin sur la bataille d'Héliopolis et la situation actuelle de l'armée française. "Comment, dit le Premier consul, avez-vous pu, vous, Desaix, attacher votre nom à la capitulation d'El-A'rych ?" - Je l'ai fait, répondit Desaix ; je le ferai encore, parce que le général en chef ne voulait plus rester en Égypte, et que, dans une armée éloignée et hors de l'influence du gouvernement, les dispositions du général en chef équivalent à celles des cinq sixièmes de l'armée. J'ai toujours eu le plus grand mépris pour l'armée du grand vizir, que j'ai observée de près ; j'ai écrit à Kleber que je me faisais fort de la repousser avec ma seule division. Si vous m'aviez laissé le commandement de l'armée d'Égypte et que vous eussiez emmené Kleber, je vous aurait conservé cette belle province, et vous n'eussiez jamais entendu parler de capitulation. Mais enfin les choses ont bien tourné, et Kleber, à Héliopolis, a réparé les fautes qu'il avait faites depuis six mois $»^{6}$.

Desaix répète en réalité des explications suggérées auparavant dans plusieurs lettres envoyées vers l'Italie, dans lesquelles il prenait les devants en prévision du courroux de Bonaparte. L'une, d'Alexandrie le 21 février 1800, paraît très explicite sur ses remords :

«L'évacuation de l'Égypte est signée, mon général, vous serez certainement surpris, surtout de ce qu'elle l'est par moi qui me suis toujours prononcé pour la conservation de cette importante conquête. Vous le serez moins quand vous connaîtrez les circonstances où je me suis trouvé. Je vous assure que je n'ai rien épargné pour vous donner le temps d'y envoyer des secours et que je n'ai obéi qu'à l'ordre très précis du général en chef. Vous m'avez donné l'ordre de vous rejoindre dans le courant de l'hiver [...]. Personne ne vous est plus dévoué que moi. Personne n'a plus envie d'être utile à votre gloire $»^{7}$.

4 Kléber, Desaix : le sort des deux hommes est intimement lié dans le parcours et la mémoire de Bonaparte. N'avait-il pas prévu en 1798, au grand dam du Directoire qui se méfiait des anciens amis de Moreau, de les laisser en France si jamais reprenait la 
guerre continentale? La campagne d'Égypte pour lui achevée, le républicanisme de Kléber, son indépendance d'esprit et de ton l'avaient persuadé de le mettre devant ses responsabilités en lui confiant une armée en piteux état matériel et moral. Entre le nouveau commandant et Desaix, mieux en cour auprès du Corse, l'affrontement avait été larvé. Confiantes depuis le siège de Mayence, les relations entre les deux soldats que quinze ans séparaient étaient également complexes, partagées entre une solidarité admirative et une compétition amicale: Kléber, l'aîné, avait réduit le pouvoir des commandants des provinces égyptiennes, diminué la circonscription sous contrôle de Desaix (abandonnant la moyenne Égypte à Friant) avant de le rappeler auprès de lui en octobre 1799 pour préparer la bataille contre le Grand Vizir; il l'avait installé à ses côtés et à ceux de son collègue Reynier parmi les membres de l'Institut du Caire, en contradiction avec les vœux de son prédécesseur qui avait exclu ses généraux de cette enceinte, les jugeant plus utiles sur le front des troupes; il l'avait enfin compromis dans les négociations avec un ennemi ottoman que Desaix était certain de pouvoir vaincre ${ }^{8}$. Mais dans les Campagnes d'Égypte et de Syrie, Napoléon s'attache exclusivement à deux portraits moraux antinomiques. Ce sont les seuls auxquels il s'appliquera vraiment dans une brève présentation de ses anciens généraux :

«Desaix était l'officier le plus distingué de l'armée ; actif; éclairé, aimant la gloire pour elle-même. Il était d'une petite taille, d'un extérieur peu prévenant, mais capable à la fois de combiner une opération et de la conduire dans les détails d'exécution. Il pouvait commander une armée comme une avant-garde. La nature lui avait assigné un rôle distingué, soit dans la guerre, soit dans l'état-civil. Il eût su gouverner une province aussi bien que la conquérir ou la défendre.

Kleber était le plus bel homme de l'armée. Il en était le Nestor; il était âgé de quarante-cinq ans. Il avait l'accent et les mœurs allemandes [...]. Kleber avait dans le caractère on ne sait quoi de nonchalant qui le rendait facilement dupe des intrigants. Il avait des favoris. Il aimait la gloire comme le chemin des jouissances. Il était homme d'esprit, de courage, savait la guerre, était capable de grandes choses, mais seulement lorsqu'il y était forcé par la nécessité des circonstances "».

5 Une telle figure d'officier républicain ne pouvait échapper aux artistes de l'expédition. Pour la postérité, Dutertre, membre de l'Institut d'Égypte, a dessiné un portrait en pied de Desaix, une pose hiératique et académique qui insiste davantage sur le rôle d'administrateur de son modèle : la veste de son uniforme déboutonnée, le général a une main dans la poche, l'autre sur l'estomac; en arrière-plan et en plongée, de solides fortifications, de pauvres habitations en terre battue, une population variée qui vaque à ses occupations quotidiennes. Denon, seul peintre à l'accompagner d'Assyût à l'oasis du Fayyûm, puis de Dendara à Lûqsor, construira de son vivant l'image du «sultan juste» en magnifiant par un dessin "la clémence de Desaix». Rendant la justice sous un palmier, son héros, en grand uniforme, ouvre les mains au jeune autochtone qu'il doit condamner - où l'on mesure ce que le panégyrique emprunte à l'imagerie royale française... Ce coupable sauvera sa tête mais sera fustigé de trente coups de fouet selon le général Belliard ${ }^{10}$; une peine oubliée dans la relation faite par Denon :

«À Miniel-Guidi [...] pendant que nous attendions assis à l'ombre, on amena au général Desaix un criminel. On criait, c'est un voleur: il a volé des fusils aux volontaires, on l'a pris sur le fait; et nous vîmes un enfant de douze ans, beau comme un ange, blessé au bras d'un coup de sabre; il regardait sa blessure sans émotion; il se présenta d'un air naïf et confiant au général, qu'il reconnut aussitôt pour son juge. ô puissance de la grâce naïve ! pas un assistant n'avait conservé de colère. On lui demanda qui lui avait dit de voler ces fusils: Personne; qui l'avait porté à ce vol : il ne savait [...]; s'il avait des parents : une mère, bien pauvre et 
aveugle ; le général lui dit que s'il avouait qui l'avait envoyé, on ne lui ferait rien ; que s'il s'obstinait à se taire, il allait être puni comme il le méritait : Je vous l'ai dit, personne ne m'a envoyé, Dieu seul m'a inspiré ; puis mettant son bonnet aux pieds du général : Voilà ma tête, faites-la couper [...]. Pauvre petit malheureux! dit le général ; qu'on le renvoie [...]. Il partit avec le sourire de la confiance ${ }^{11}$. fréquemment le logement, l'aide plus que Desaix dans ses relevés artistiques et scientifiques, il voue au second une admiration sans bornes, fondée sur les conversations privées, les dangers et les découvertes librement et longuement partagés au milieu d'infinies souffrances lors de la conquête de la haute Égypte ${ }^{12}$. «J'ai trouvé dans le général Desaix un savant, un curieux, un ami des arts ; j'en ai obtenu toutes les complaisances que pouvaient lui permettre les circonstances", se réjouira-t-il ${ }^{13}$. Lecteurs des Mille et une nuits, les deux hommes apprécient les conteurs orientaux dont la «mémoire prodigieuse » du général conserve les récits. Se voulant dans le même temps héritiers et porteurs des Lumières, sûrs de leur culture, ils se heurtent, à l'instar des savants français au Caire, à une vision différente de l'univers qu'aucune démonstration raisonnée ne vient ébranler. Ainsi l'officier, interrogeant un homme de loi sur l'origine du tonnerre, s'entend répondre que tout cela vient d'un ange. «On est toujours émerveillé, conclut Denon, d'entendre un homme sensé, avec une barbe vénérable, faire un conte aussi puéril. Desaix voulut lui expliquer différemment ce phénomène mais il trouva son explication si inférieure à la sienne qu'il ne prit pas même la peine de l'écouter ${ }^{14}$. L'abondance de curiosités nourrit les dialogues et les élucubrations des deux Français, depuis les crocodiles du Nil, sur lesquels ils dissertent le 26 janvier 1799, jusqu'aux vestiges historiques les plus prestigieux (le général, qui a admiré ceux qui embellissent Rome, rêve de ramener dans sa patrie les obélisques de Lûqsor ${ }^{15}$; il s'essaie à interpréter la datation du Zodiaque de Tentiris, s'extasie devant les temples de Philae ou de Thèbes). Cet éclectisme ne doit cependant pas faire illusion sur leurs capacités à embrasser le monde. Denon ne se prive pas, parce que légèreté, naïveté et simplicité nourrissent selon lui le vrai, de pointer la candeur peu éclairée de Desaix lorsqu'il se laisse saisir devant Oxyrinchus par la mélancolie des ruines et l'oppression du désert :

«Mon ami, me dit-il, ceci n'est-il point une erreur de la nature? Rien n'y reçoit la vie; tout semble être là pour attrister ou épouvanter; il semble que la Providence, après avoir pourvu abondamment les trois autres parties du monde, a manqué tout à coup d'un élément lorsqu'elle voulut fabriquer celle-ci, et que, ne sachant plus comment faire, elle l'abandonna sans l'achever.

- N'est-ce pas plutôt, lui dis-je, la décrépitude de la partie du monde la plus anciennement habitée? Ne serait-ce pas l'abus qu'en auraient fait les hommes qui l'a réduite en cet état. Dans ce désert, il y a des vallées, des bois pétrifiés; il y a donc eu des rivières, des forêts : ces dernières auront été détruites; dès lors plus de rosée, plus de brouillards, plus de pluie, plus de rivières, plus de vie, plus rien ${ }^{16}$.

Gammes savantes qui montreraient les limites des enseignements fondamentaux d'Effiat si on ne les sentait en partie recomposées pour la plus grande gloire d'un Denon soucieux de postérité, elles sont aussi les conséquences des invitations de l'Encyclopédie. "C'était jadis un pays d'admiration, c'en est un aujourd'hui à étudier ", proclame celleci dans son article "Égypte», preuve d'une fascination pour l'ancienneté d'une civilisation et d'une volonté de faire resurgir cette dernière, magnifiée par David dans les fêtes révolutionnaires. L'abolition du despotisme ottoman et du régime des mamelouks, facteurs de dégénérescence d'un Orient autrefois brillant et maintenant décadent, l'exportation des progrès intellectuels, scientifiques et techniques d'un 
Occident perçu comme désormais supérieur en sont les moyens : les proclamations de Bonaparte (celle du 27 juin 1798 particulièrement), la fondation, le 22 août 1798 au Caire, de l'Institut "pour les sciences et les arts" en attestent ${ }^{17}$. Denon et Desaix assument et servent cette politique, se pensant absolument autres que les autochtones qu'ils observent et qu'ils jugent ${ }^{18}$ - et que les Français choquent par leurs mœurs et leur licence. Et l'Auvergnat, « très noir et très sec Égyptien qui, au milieu du désert brûlant et à côté de la peste " se rappelle au bon souvenir de sa cousine adorée ${ }^{19}$, goûte comme ses hommes à la luxure, moins seul dans ses chevauchées que ne le rapportent ses thuriféraires :

«Je vous dirai un mot de mes amours. J'ai aimé la jeune Astiza, gentille Géorgienne, belle comme Vénus, blonde et douce. Elle avait quatorze ans, deux boutons de rose ; elle m'appartenait de succession comme souverain du pays, son maître étant mort. Je pouvais la vendre six mille livres. Je l'ai donnée, je la craignais. J'ai reçu en présent Sarah, Abysienne folâtre, gaie, âgée de quinze ans; elle était la compagne de mes voyages. J'ai possédé Mara, naïve enfant du Tigre. J'avais encore Fatma, grande, belle, bien formée, bien malheureuse. Imaginez qu'au milieu de mon sérail, témoin de mes plaisirs, de mes jouissances, de mes voluptés, elle ne pouvait les partager [...]. Voilà tout ce qui composait mon sérail. Accompagnez-le de trois négresses, vous connaissez tout mon ménage. Joignez à cela un petit nègre, Baquil, un petit mamelouk, Ismaël, beau comme un ange, vous avez la composition de ma maison. Voyez à présent mes aides de camp cherchant à y pénétrer, mes eunuques les repoussant, moi couché mollement sur de beaux coussins, me faisant servir par quelques-unes de ces femmes, tantôt l'une m'endormant voluptueusement et me frottant doucement les pieds, tantôt l'autre me déshabillant, faisant ma toilette avec une légèreté admirable, un soin inconnu à l'Europe $[. ..] »^{20}$.

8 La "mission civilisatrice " - selon une terminologie largement imaginée à partir de l'Esquisse d'un tableau historique de l'esprit humain de Condorcet -, la capacité d'adaptation aux mœurs, aux cultes et aux rites des autochtones ne sauraient servir de justification. À l'inverse, l'empreinte du mythe aide sans doute à ces reconstructions sociales fort éloignées des idéaux de liberté individuelle défendus au même moment par le législateur français, quoique ce dernier n'étende pas jusqu'aux femmes leur champ d'application. Desaix s'inscrit dans une tradition guerrière qui, à l'heure où perdurent les batailles, valorise peu le citoyen et autorise le tribut du vainqueur, une manière de conquérir difficilement conciliable avec le discours sur la régénération. Ce qui, en langage militaire, est bien traduit dans ses ordres au général Friant :

" La grande affaire est de presser l'organisation du pays, de chasser constamment et détruire Mourad, soumettre vos méchants Arabes, prendre des otages en très grand nombre de tous ceux qui voudront être en paix [...]. Mettez bien vos commandants de province en mouvement; ils croient avoir tout fait quand ils sont restés dans leurs capitales, occupés à des constructions. Il vaut bien mieux courir le pays. Si on a cent hommes, on n'est pas plus sûr dans une ville que dans un village. En marchant beaucoup et rapidement, on fait trembler tout le pays, on le tient en respect [...]. Cet imbécile de Mourad, qui se tourmente sans cesse, croit que les Turcs le protégeront; il ne sait pas qu'ils ont juré sa mort. Il ne comprend pas ses intérêts $»^{21}$.

9 L'adversaire n'est guère ménagé, quoi qu'aient pu en dire ultérieurement biographes et historiens ${ }^{22}$. En son nom est condamné son insaisissable chef, qui ne cesse de réchapper des encerclements français. Indissociables des combats, l'occupation et l'administration des territoires que Desaix soumet à sa férule lui donnent l'occasion d'un effort de rationalité largement fondé sur la méconnaissance persistante des habitants, quelles que soient les synthèses sur les tribus, tentées pour la pallier. Tout paraît à faire 
concernant les mamelouks: «Ces arabes que l'on nous a représenté les maitres de l'Égypte ont été fort peu connus par les voyageurs. Les idées qu'ils nous en ont données sont peu justes. C'est très pardonnable. Le moyen de bien les voir est bien difficile et leur situation varie trop pour qu'on les puisse bien voir sans y employer un temps infini.» Desaix simplifie pourtant aussitôt, opposant aux précédents un peuple de fellahs, qu'il veut par tradition pasteurs et voleurs, vivant en tribus essentiellement familiales - leurs noms insistent sur la dépendance au père, chaque chef devant sa puissance à sa progéniture, - et sur les liens fraternels entre clans ${ }^{23}$. Le tout justifie un recueil incomplet de Notes sur les diverses tribus arabes de l'Égypte ${ }^{24}$, soit une trentaine d'entrées dont onze seulement donnent lieu à des développements substantiels, quatre autres se contentant d'une mention, bien souvent le nom du chef militaire de l'entité considérée. Car l'intérêt de ce travail est d'abord stratégique avant de se vouloir historique ou ethnologique ${ }^{25}$, deux soucis largement balayés par la maladroite synthèse de départ :

«Il existe en Égypte une grande quantité de tribus diverses connues sous le nom d'arabes. Elles doivent en partie leur origine a des tribus d'habitans de l'intérieur de l'Afrique qui sont venues en Égypte soit chassées par d'autres plus puissantes, soit attirées par des terres pour les bestiaux soit aussi amenées par les bey dans les différentes guerres soit enfin par d'autres tribus qui pour [...] y ont attiré des tributs nouvelles pour les soutenir et pour les défendre. Ce qui distingue les arabes les plus proprement ainsi nommés c'est qu'ils habitent des tentes et qu'ils vivent en grande partie de leurs bestiaux. Ils ont cependant des terres et souvent leurs chefs en ont beaucoup. Ils sont riches. Les tributs d'arabes venus de l'intérieur de l'Afrique sont aisé a connoître a leur costume qui est différent de celui des habitans. L'habit blanc comme la neige qui n'est pas une tunique faite comme une chemise mais une grande pièce d'étoffe qui les enveloppe. Ils portent habituellement encore une grande pièce d'étoffe qui recouvre leur tête et descend jusqu'en bas a leur pied. Les tribus se sont en général établies sur la rive occidentale du fleuve depuis Alexandrie jusqu'à Siout. Plus haut il n'en existe plus ».

Alors défilent les ombres imprécises des hommes venus de Tripoli il y a huit décennies (les Tenatris) ou deux ans (les Nozaratis), des Tunisiens de Mohamed Aboudabal, répartis depuis soixante-dix ans au moins en huit dizaines de villages agricoles, des Aladis, trois mille individus, caravaniers et propriétaires de dromadaires divisés en quatre tribus réparties le long du Nil et dirigées par « un jeune homme enfant » dont le père «étoit très méchant" - "leur origine est perdue, ils n'ont plus de condition et se disent enfans du diable ». Sans compter deux cents éleveurs de buffles et cultivateurs d'orge conduits par Mohamed Abou Achmed, dormant sous la tente et accompagnant si besoin les caravanes ; les Attoumieh Maasis, éléveurs chassés du mont Sinaï et vivant désormais de ce que leur payent les groupes de pèlerins sur le chemin de la Mecque; les Alezak Elegate, de même origine géographique, cultivateurs-éleveurs allant jusqu'au fin fond de l'Afrique chercher des esclaves, si nombreux et sur tant de sites d'occupation, jusqu'aux îles du Nil, qu'ils ont fini par parler trois dialectes. Leur puissance militaire, reposant sur une cavalerie de trois cents d'entre eux, est telle qu'ils contrôlent, depuis six ans et un grand combat, les escortes de caravanes. Plus rares semblent les sédentaires: les Bescharis, entre Nil et mer Rouge, dans ces "effroyables déserts", vivant du millet et des chevaux et dromadaires qu'ils échangent contre des toiles; au niveau de la première cataracte du fleuve, les hommes de Soliman Cachef, protégés par une impressionnante forteresse "garnie de canons" passant pour imprenable, exportateurs de dattes et de céréales. 
11 Suivent les descriptions de plusieurs villes, particulièrement Akhmîm et Girga. Desaix égraine la carte des productions textiles : les châles et turbans du Fayyûm, la laine et le coton de Beni Suef, la soie d'Asyût (échangée avec les caravanes venues d'Afrique noire), les fabriques de Jaffa ou de Farshût. D'Akhmîm, anciennement soutenue par Constantinople, le Français retient le relatif délabrement économique, l'occupation par les mamelouks ayant pour un temps anéanti les ateliers et l'agriculture périurbaine, qui contribue pourtant à nourrir mille familles. Demeurent d'une époque plus brillante cent cinquante familles d'esclaves noirs, désormais établis comme petits boutiquiers, tandis que perdurent de grosses manufactures textiles et le commerce de gommes, pavots et coton, qui encourage le trafic avec la Nubie et l'Abyssinie et fait prospérer sur place auberges et activités judiciaires. L'observateur est plus prolixe sur Girga. Capitale de la haute Égypte, elle se remet lentement de la peste qui l'a ravagée une vingtaine d'années auparavant. "Aujourd'hui il n'y a plus que 1600 familles dont 1200 musulmans, 300 coptes schismatiques, 100 catholiques», note-t-il, insistant sur l'importance des fonctions religieuses attachées aux mosquées (quinze petites et neuf grandes), puis sur le poids des quatre-vingts hommes de loi (les ulémas), parmi lesquels on trouve les plus grosses fortunes - 1200 livres par an au moins, estime le général, seul le cheikh Abduselmensur pouvant compter sur plus de 5000 , tandis que le couvreur de toit se contente de 6 à 8 sols par jour, le maçon de 8 à 9 . Les commerces à l'évidence arrêtent le promeneur, comme autant de douceurs offertes au soldat harassé : «il y a à Girgé 300 boutiques de marchands de toutes espèces dont 15 de barbiers, 15 de caffé, un 100 de marchands d'épicerie, droguistes, tabac et 30 de cordonniers, un 30 de familles d'ouvriers en selles et cuirs ", auxquels s'ajoutent les employés des bains, les armuriers, les commerçants en tissus et en toiles, une centaine de tisserands, trois cents familles d'ouvriers, près de deux mille agriculteurs, quatre cents domestiques, porteurs d'eau ou pauvres hères. Les petits métiers gagnent en indépendance avec l'occupation française :

«Dans les temps des mamelouks les professions différentes ressortissoit aux différents domestiques des commandants, par exemple les caffetiers, les meuniers, les loueurs d'ânes, les marchands de jus de réglisse dependoit de celui qui tond la tête du bey. Les faiseurs d'huile aussi [...]. [Le domestique] etoit obligé de les surveiller, il avoit droit de police et faisoit bastonner ceux qui étoient en faute. Celui qui étoit à la tête des cuisines surveilloit les bouchers, les patissiers, les petits cuisiniers ».

12 Les minorités religieuses ont su faire leur place: catholiques ou coptes sont très présents (administrateurs, officiers des finances, marchands de coton, ou bien ouvriers des ateliers textiles). Desaix s'associe un représentant de la deuxième communauté, Ya'qoub, ancien intendant de Sulayman Bey. C'est lui qui va prendre à sa charge l'organisation financière de l'expédition, jouant sur sa connaissance parfaite des mécanismes fiscaux égyptiens et n'oubliant pas de se servir au passage ; il participera de surcroît à la mise sur pied d'un service de renseignements espionnant efficacement les mouvements des mamelouks; en bref, un personnage puissant, regardé par les populations, selon les récits de Belliard, comme le Grand Sultan ${ }^{26}$.

$13 \mathrm{Si}$, dans sa marche forcée et lors de ses combats, Desaix ne ménage pas ses hommes ${ }^{27}$, beaucoup moins soucieux de les protéger que Kléber, il ne s'éloigne pas non plus de la première déclaration de Bonaparte en terre égyptienne, le 24 juillet 1798, promettant aux villages récalcitrants ratissages réguliers, massacres et incendies. Faisant face dans le Fayyûm à la guérilla des mamelouks, qui font courir des risques immenses aux 
paysans qu'ils contrôlent puis abandonnent, le Français n'hésite pas à faire des exemples, détruisant ainsi, pour assurer la tranquillité des communications et mater plusieurs rebellions, les villages de Cercini et de Tahta (dans ce dernier, les maisons sont incendiées et une partie de la population massacrée le 21 nivôse an VII [10 janvier 1799]). "Je regarde l'armée en Égypte invincible et inattaquable», proclame-t-il fièrement en considérant particulièrement les quatre mille cavaliers qu'il souhaite lui voir compter ${ }^{28}$. Afin d'en assurer le recrutement et la formation, il prône un amalgame entre les nationalités, auxquelles seraient enseignés le français et l'arabe, afin de faciliter une complicité fraternelle; entre les pratiques des cavaleries mamelouk et française étudiées dans des écoles où se mêleraient les jeunes gens. Il plaide en faveur de mesures attractives pour les nouvelles recrues: mousses de plus de treize ans stationnés dans la rade du Caire («Rien n'est intrépide comme un enfant de quatorze ans. Il est capable de tout apprendre avec vivacité et ne connaissant pas le danger il est capable de l'affronter de la manière la plus brave »), domestiques de l'armée occupante, mamelouks adolescents (il en espère deux mille), prisonniers faits sur les Turcs (en leur promettant famille et propriété s'ils n'ont ni l'une ni l'autre), Noirs achetés ou échangés en grand nombre contre des grains aux caravanes de la haute Égypte, enfants brutalement enlevés à leurs familles révoltées contre l'occupant enfin ${ }^{29}$. Afin de détourner les plus cultivés des carrières administratives mieux rémunérées, il faudrait selon Desaix augmenter les soldes, assouplir la discipline, offrir promotions et commandements de bataillons ethniquement homogènes à l'issue de trois mois d'instruction. Ceux-ci se concluraient par un concours d'excellence sous l'arbitrage du général en chef, primé par un armement complet de mamelouk - où l'on voit combien les réminiscences de l'histoire romaine et les principes de l'école d'Effiat continuent de nourrir l'imagination du général... Ainsi, une fois les Français de retour au pays, demeureraient dans les territoires conquis, grâce à tous ces efforts d'émulation, «des usages, des habitudes et un goût de la langue qui ne pourrai[en]t qu'être très utiles pour la nation ».

Lorsque le soldat gouverne la moyenne et la haute Égypte, il s'interroge sur les lendemains de la conquête : «Sera-ce comme les Espagnols et les Russes gouvernant les premiers l'Amérique, les seconds la Sibérie, en détruisant la population par des travaux énormes ? Sera-ce en distribuant les propriétés et les habitans à des Français qui auront sur les paysans les droits que la féodalité donnait à nos seigneurs sur leurs vasseaux $»^{30}$ ? Desaix souhaiterait accorder immédiatement aux Égyptiens la citoyenneté française, mais la réalité telle qu'il la perçoit le convainc de surseoir et lui fait justifier, selon une contradiction majeure inhérente à toutes les guerres de la Révolution et par un procédé discursif souvent employé depuis 1792, une colonisation contraignante :

«Comment faire entendre ses droits à une nation flétrie par le despotisme depuis des siècles, à des hommes qui n'entendent point notre langue et qui nous haïssent par préjugé ? Je crois qu'il vaut mieux faire de ces droits et de ces privilèges une récompense pour l'habitant qui aura pris notre manière d'être et qui se sera dévoué aux Français. Mais avant de donner des loix à un pays, il faut commencer par en être bien le maître $»^{31}$.

Plus que le paiement de contributions, dont Desaix juge qu'il a jusqu'à présent par trop obnubilé les conquérants, police et justice deviennent alors les pierres angulaires de la politique d'occupation ${ }^{32}$. La première devrait être confiée à un administrateur général secondé dans chaque province par des subordonnés sous le contrôle de l'armée (tous 
seraient à l'origine officiers), chargés de tenir un registre de renseignement sur chaque village, de poursuivre "voleurs et méchants par tous les moyens ", ce qui suppose qu'ils aient une cavalerie de dromadaires et droit de guerre et de paix pour jouer sur les rivalités tribales afin d'arriver à leurs fins - des primes leur seraient allouées à chaque arrestation. Des interprètes auraient pour double mission de comprendre l'origine des haines et des conflits villageois et de tenter selon les cas de les apaiser, par exemple en aidant à lier des clans ennemis par des mariages ${ }^{33}$, ou de les attiser. Pourquoi, d'ailleurs, ne pas "avoir des otages de toutes les tribus" au Caire, des femmes et surtout des enfants éduqués dans des écoles spéciales ( les Anglais et les Hollandais ne gouvernent les Indes que par ce moyen») ? À toute rébellion correspondrait une ponction supplémentaire sans espoir de reconstituer les familles ainsi déchirées («il faut détruire les races méchantes »). Un code pénal imprimé, très simple, fixerait les récompenses pour celui qui livrerait les coupables - avec une gradation selon que la victime serait française ou arabe, selon qu'il s'agirait d'un meurtre ou d'un vol -, les punitions, pour qui protégerait voleurs et assassins, et les peines encourues. La mort est promise au détrousseur, douze livres le sont au contraire à l'assassin d'un guerrier arabe... Pensant aux soldats détachés de leur colonne, proies faciles pour les mamelouks, leur commandant propose "que la mort d'un Français soit extrêmement vengée » : l'arrestation des cheikhs des villages voisins du drame, une amende sous huit jours en fusils ou en argent, puis une autre de semaine en semaine tant que les coupables ne seraient pas dénoncés. Les familles de ces derniers, à moins que les dénonciateurs n'en soient, seraient incarcérées, leurs maisons brûlées, les complices et les protecteurs du prévenu mis à mort. Quant au meurtrier lui-même :

«Je voudrais que le coupable fut pendu à un arbre avec ordre de l'y laisser, et cela dans le lieu du crime.

Il faudrait des supplices pour les grands crimes. Les habitans ne craignent pas la mort, ils craignent la douleur.

Dans les préjugés du pays, la famille du mort doit venger son sang. Il faut donc s'emparer de ses enfants et les élever dans un autre préjugé.

J'éteindrais la famille par la mort du père, la femme et les enfans $»^{34}$.

16 Il y a, dans cet énoncé des flétrissures infligées, bien éloigné des enseignements de Beccaria, contradiction évidente avec l'image pacifiée et généreuse du "sultan juste " imaginée par Denon mais aussi avec les lettres particulières de Desaix, épouvanté par la cruauté des peines égyptiennes - pour les fautes privées il est vrai : la mort décidée par un père pour sa fille délurée, les yeux crevés de l'amant polygame dont les maîtresses sont noyées ${ }^{35}$.

Dans la réalité, le général cherche appui auprès des cheikhs de village. Les réunissant en assemblées pour discuter de problèmes du gouvernement, de la production agricole, il s'interpose aussi dans les conflits villageois, tel que le rapporte Belliard dans son Journal : «Le général Desaix a réuni les cheiks; chacun a déduit ses raisons ; on a pesé le pour et le contre dans la balance de la justice, et des hommes, qui une demi-heure avant voulaient se détruire, ont fini par goûter les sages réflexions, les avis ou les ordres du conquérant et se sont retirés bons amis ${ }^{36}$. Sa préoccupation va tout autant à la santé de ses administrés, auprès desquels il envoie les médecins de son armée, et au développement économique de ces régions où les produits du petit élevage paraissent abonder à bas prix. Ce développement lui paraît devoir être le fruit d'une politique de sédentarisation forcée qui, liée au souci de police plus haut évoqué, utilise pour le coup l'arme de l'impôt. Celui-ci frappera chevaux et chameaux («ils sont très à charge, ne 
travaillent pas, ils ne servent à l'habitant que pour faire la guerre ») : neuf livres annuelles pour un cheval ou une jument, avec des exemptions pour celle qui aura mis bas un mulet, pour un animal de labour, de moulin ou de trait, pour un chameau porteur. Par des primes et la suppression du mîrî (la contribution foncière) sur les terres ruinées par les combats, les cultures céréalières ou celles des dattiers, les jardins, les enclosures sont au contraire encouragés. En bref, « la tribu d'Arabes qui sera tout à fait pasteur, on les fera soumettre à des impôts. Celle qui cultivera le sucre payera peu. On les attachera à la culture par toute sorte d'encouragements ". L'idée majeure, importée de France, est d'établir un peuple de propriétaires, "le plus propriétaire possible ", attaché à ses habitations et à leur embellissement - un peuple qui comptera aussi selon le plan de Desaix des colons défricheurs, des soldats de l'armée française d'origine égyptienne récompensés par des terres héréditaires hors la juridiction des cheikhs $^{37}$. Sont construits des meuneries, des chenaux d'irrigation, des routes autour de Qinâ et des aménagements portuaires à Qusayr (détruits par les Anglais à la mi-août 1799). Cette politique est complétée par des aides au commerce du blé, du café et des indiennes, par un accueil privilégié aux riches pèlerins et à la caravane annuelle du Dar-Four (de palabres en cadeaux échangés, Desaix au titre de ces derniers reçoit le jeune Baquil qui le suivra à Marengo).

18 Après un retour d'Égypte difficile (interrompu grossièrement par les Anglais de lord Keith d'abord, les Barbaresques ensuite, ralenti enfin par l'obligatoire mise en quarantaine), Desaix est bien loin de songer à une quelconque gloire. Ce n'est ni l'amoureux des Lumières ni l'administrateur qui se découvre dans ses correspondances privées mais un soldat impatient d'en découdre, savourant par avance l'art de la guerre, quelle que soit la place qui lui permettra de l'exercer. Ainsi l'exprime-t-il en mai 1800, depuis son lazaret de Toulon, à son ami Clarke - avec la perspective de comprimer toute velléité d'invasion des Anglais (« les rosser et de là courir au plus chaud »), il pense alors rejoindre l'armée du Rhin :

«Il faut tout faire pour finir promptement la guerre et je veux en être. Je vous assure qu'il m'est égal d'être ce qu'on voudra, tous les grades me sont égaux; je tremble qu'on m'en donne de trop élevé, vous savez que je ne les aime pas. Je vous dirai confidentiellement que d'être à une armée bien active et en bon état serait mon bonheur; en ce moment, je suis un peu fatigué pour aller à une armée désorganisée $»^{38}$.

19 Fait-il allusion à l'armée d'Italie ? Le même jour en tout cas, il présente à Bonaparte une candidature comparable pour les champs de bataille allemands, dans un style plus laborieux tant est grande la nécessité de se justifier avant même la rencontre de Montebello. Pour se faire pardonner de n'avoir pas rejoint dès l'hiver 1799 le Premier consul qui le lui demandait, Desaix fait un peu crédible aveu d'impuissance ( Le général Kleber n'a jamais voulu y consentir il m'a retenu et malgré moi m'a fait conclure la convention de el arich») puis déroule le récit de son périple chaotique depuis l'Égypte jusqu'à la France. S'ensuit l'assurance d'une sujétion totale dont son interlocuteur voudra en Italie des preuves supplémentaires : «Travailler à augmenter la gloire de la république, la votre, est tout mon désir [...]. Je sais que vous voulez porter la France a son plus haut point de gloire et cela en rendant tout le monde heureux; peut on faire mieux $»^{39}$ ? Le compliment et la question, d'une ingénuité politique évidente, trouvent d'une certaine façon réponse sous le feu de Marengo. égyptienne. Elle n'est pas seulement inscrite sur les propylées de Philaé, aujourd'hui 
englouties sous les eaux du barrage d'Aswân ${ }^{40}$. Elle inspire d'anciens compagnons admiratifs, de l'adjudant-général Mellinet aîné, auteur dès 1800 d'un Chant sur la mort de Desaix ${ }^{41}$, à Ya'qoub, publiant une élégie aux mânes de celui qu'il avait servi (le véritable auteur en serait son ami Lascaris, chevalier de Malte). Sur l'olympe, le dignitaire copte place Desaix sans doute trop près d'un futur empereur dont le culte pour son ancien subordonné sera prudemment orchestré. Il réitère d'une certaine façon son acte d'allégeance, dévoilant combien il est conquis par le modèle économique des vainqueurs d'un jour et bien près de les considérer ainsi qu'ils se voulaient, les porteurs d'un savoir autrefois oriental :

« Tu vivras éternellement dans la postérité et dans ma reconnaissance, déjà ton ombre chérie voltige dans l'immensité de l'espace avec celle des héros de l'Antiquité, déjà elle s'y unit au génie tutélaire de Bonaparte, et de cette union si redoutable aux ennemis et aux peuples de la terre va naître la prospérité et la civilisation de l'Orient $»^{42}$.

21 Sur le terrain, le général a appliqué sans défaillir les principes énoncés au Caire par les membres de l'Institut, principes qui participent à la fois du sentiment de supériorité précédemment évoqué et d'un état d'esprit qui soude les conquérants autour des idéaux de progrès et de philanthropie. Desaix par ses décisions ne contredit pas Desgenettes, proposant un enseignement médical à destination des Égyptiens, pas plus qu'il ne contrarie les rêves de Volney évoquant dans le Courrier de l'Égypte ce que pourrait être l'amalgame des peuples, la nation construite par Bonaparte grâce à « des collèges militaires où les jeunes gens français, coptes, arabes, s'enseignent mutuellement l'arabe, le français, la géographie, les mathématiques, les sciences exactes $»^{43}$. Homme de culture et de raison - avec les limites imposées à son éclectisme par son jeune âge et sa carrière militaire tôt remplie -, curieux du progrès scientifique et technique, patriote convaincu des bienfaits civilisateurs d'une colonisation à marche forcée et classant en catégories simplistes les peuples rencontrés, Desaix, après avoir marché dans les pas de César, pouvait-il être absent des manuels scolaires de la République des Jules?

\section{NOTES}

1.A. Ehrard et G. Tisserand, Louis Charles Antoine Desaix, général, catalogue de la Conservation des musées d'art de la ville de Clermont-Ferrand, Clermont-Ferrand, 1983.

2.Service historique de l'Armée de Terre, 1 M 513 et 514. L'orthographe de Desaix, souvent incertaine, sera systématiquement respectée dans les citations qui suivent extraites de ces carnets (comme pour celles issues de sa correspondance).

3.J.-P. Doguereau, Journal de l'expédition d'Égypte, Paris, 1997.

4.J. Delmas et P. Lesouef, « Napoléon chef de guerre : les années heureuses

(1789-1809) », in Napoléon Bonaparte. L'ouvre et l'histoire (sous la direction de J. Massin),

Paris, 1970, p. 22. Lettre de Bonaparte à Desaix, Le Caire, 11 août 1799 : «J'ai été peu satisfait, Citoyen Général, de toutes vos opérations pendant le mouvement qui vient 
d'avoir lieu. Vous avez reçu l'ordre de vous porter au Caire, et vous n'en avez rien fait. Tous les événements qui peuvent survenir ne doivent jamais empêcher un militaire d'obéir ; et le talent, à la guerre, consiste à lever les difficultés qui peuvent rendre difficile une opération, et non pas à la faire manquer. Je vous dit ceci pour l'avenir. » 5.Cité par A. Sauzet, Desaix, le «sultan juste», Paris, 1954, p. 229.

6.Mémoires pour servir à l'histoire de France sous le règne de Napoléon Ier, dans Bonaparte. L'œuvre et l'histoire, op. cit. note précédente, p. 241.

7.Cité par A. Sauzet, op. cit. note 5, p. 260.

8.H. Laurens, L'expédition d'Égypte (1798-1801), Paris, 1997, pp. 316 et sqtes / A. Sauzet, op. cit. note 5, p. 258. Kléber, dans une lettre du 27 janvier 1800 à Desaix, montre combien il est conscient de l'épreuve infligée à son subordonné : «Je conviens, mon cher général, vous avoir donné une mission d'autant plus cruelle que le succès dont vous l'avez couronnée ne peut vous faire espérer d'autres récompenses que celle de votre propre satisfaction et la mienne. Une œuvre de raison a toujours été accueillie avec indifférence et du public et du gouvernement, quoique pour l'accomplir, il ait fallu plus de lumière, de talent et de persévérance que pour l'action la plus brillante en apparence [...]. Enfin, cher général, pour dissiper entièrement votre mauvaise humeur contre moi, mettez un instant Desaix à la place de Kléber et celui-ci à la place du premier et demandez alors à Desaix ce qu'en pareille circonstance il aurait fait? Mais la plus grande tâche vous reste à remplir à Paris. C'est là que vous aurez à soutenir contre la toute-puissance irritée le faible qui n'a pour auxiliaire que raison et vérité. Si vous êtes écouté, votre triomphe est certain ; si vous ne l'êtes pas, vous saurez, je pense, ainsi que moi, élever votre âme au-dessus de l'injustice. »

9.Napoléon Bonaparte, Campagnes d'Égypte et de Syrie, rééd. Imprimerie nationale, Paris, 1998, pp. 178 et 36.

10.A.D. Belliard, Histoire scientifique et militaire de l'expédition française d'Égypte, Paris, 1830-1835 ; Mémoires, Paris, 1882.

11.D. Vivant-Denon, Voyage dans la Basse et la Haute-Égypte, 1802, rééd. Paris, 1998, p. 154 12.Ibidem, p. 210. Alors qu'une opération militaire les sépare un temps à la mi-février 1799, Denon se lamente : «Ce fut un chagrin pour tous deux ; nous avions passé ensemble des moments si doux et si répétés, marchant au pas côte à côte pendant douze à quinze heures de suite ; nous ne causions pas, nous rêvions tout haut ; et souvent, après ces séances si longues, nous nous disions : combien nous aurons de choses à nous dire le reste de notre vie!»

13.Ibidem, p. 30.

14.Ibidem, p. 179.

15." Il y a, à Thèbes, deux obélisques d'une taille et d'un fini de travail qui font qu'aucun de ceux de Rome ne leur est comparable. Transportés à Paris, ils y seraient bien extraordinaires ", écrit Desaix le 21 mars 1799. Cité par J. de Metz et G. Legrain, Aux pays de Napoléon. l'Égypte, Grenoble, 1912, p. 117.

16.D. Vivant-Denon, op. cit. note 11, p. 157.

17.L'Institut se donne pour but d'étudier l'ancienne civilisation égyptienne et d'assurer « le progrès et la propagation des Lumières en Égypte ». Cf. M. L. Ortega, « La "régénération" de l'Égypte : le discours confronté au terrain ", et A. Raymond, « Les Égyptiens et les Lumières pendant l'expédition française ", in L'expédition d'Égypte, une entreprise des Lumières (1798-1801), Actes du colloque de l'Académie des inscriptions et belles-lettres et de l'académie des sciences (8, 9 et 10 juin 1998) réunis par P. Bret, Paris, 1999, pp. 93-101 et 103-117. 
18.M. Reid, « Portrait d'une population : l'Égypte vue par Denon », ibidem, pp. 207-216. 19.Lettre du 21 février 1800, d'Alexandrie, à Marguerite Victoire Le Normant de Tournehem. Citée par A. Sauzet, op. cit. note 5, p. 176.

20.Lettre à la même, août 1799. Ibidem, p. 245.

21.Bibliothèque municipale et interuniversitaire de Clermont-Ferrand, A 30683, Carnet historique et littéraire, 15 juin 1900. Lettre de Desaix à Friant, 28 vendémiaire an VIII (20 octobre 1799).

22.Cf. A. Sauzet, op. cit. note 5, p. 228 : «Ce Mourad, qu'il aime bien au fond puisqu'il le traite sympathiquement d'imbécile lorsqu'il le voit se méprendre sur ses propres intérêts "; H. Laurens, op. cit. note 8, p. 421.

23.Service historique de l'Armée de terre, 1 M 513. Notes sur l'Égypte.

24.Ibidem, 1 M 514.

25.Les rubriques prévues sont les suivantes, pour chaque tribu : origine, histoire, établissement, villages, richesse, commerce, division, liaisons, population, chevaux, hommes à pied, chameaux, alliances, parentés, guerre, ennemis, nom des cheikhs, famille de ceux-ci, emploi de la fortune.

26.H. Laurens, op. cit. note 8, pp. 222-223.

27.Selon Denon, op. cit. note 11, p. 144, les suites de la bataille de Sediman sont particulièrement douloureuses : « Pour marcher il fallait abandonner les blessés, et les abandonner était les livrer à une mort assurée ; circonstance affreuse dans toutes les guerres, et surtout dans la guerre atroce que nous faisions ! Comment donner un ordre ? Desaix, l'âme brisée, reste immobile un instant ; l'intérêt général commanda ; la voix de la nécessité couvrit les cris des malheureux et l'on marcha ».

28.Service historique de l'Armée de Terre, 1 M 513. Notes sur l'Égypte.

29.Ibidem. «Je ne connais qu'un autre moyen c'est de s'emparer de tous les enfants des villages révoltés dont on détruira les peres les armes a la main, prendre ces enfants, les conduire au Caire, les mettre dans nos écoles, leur apprendre a lire écrire les deux langues, a s'habiller comme nous (...). C'est de cette manière que les Turcs sont parvenus à cette puissance qui a étonné le monde longtemps ».

30.Ibidem.

31.Ibidem.

32.Ibidem. Desaix ne répugne pas pour se convaincre du bien-fondé de ces options à recourir aux modèles historiques autochtones : «Aly Bey n'est vénéré dans toute l'Égypte que parce que seul il fit régner partout des loix vigoureuses, que partout il fit condamner les coupables et empêcher tous les crimes. C'est un des grands bienfaits que nous devons donner aux habitans. La sécurité des routes, le désarmement général résulteront bientôt de ces mesures ".

33.Desaix pense qu'il faudrait aussi encourager les mariages entre membres des tribus rebelles et Français. Son collègue Menou en donnera personnellement l'exemple.

34.Service historique de l'Armée de Terre, 1 M 513.

35.Cf. A. Sauzet, op. cit. note 5, p. 236.

36.C. de la Jonquière, L'expédition d'Égypte, Paris, 1899-1907, vol. 3, p. 512 (Journal de Belliard).

37.Service historique de l'Armée de Terre, 1 M 513.

38. Bibliothèque municipale et interuniversitaire de Clermont-Ferrand, A 30683, Carnet historique et littéraire, 15 juin 1900. Lettre de Desaix au général Clarke, 15 floréal an VIII (5 mai 1800). Clarke dirige alors le Dépôt de la Guerre. 
39.Bibliothèque nationale, NAF 1303. Collection d'autographes de Lefèvre, II. Ff 133. Lettre de Desaix à Bonaparte du 15 floréal an VIII (5 mai 1800).

40.Desaix y avait fait graver : « L'an VI de la République le 13 messidor une armée française commandée par Bonaparte est descendue à Alexandrie. L'armée ayant mis vingt jours après les mameluks en fuite aux Pyramides, Desaix commandant la première division les a poursuivis au-delà des cataractes où il est arrivé le 13 ventôse de l'an VII (...)»

41.Almanach des Muses pour l'an IX de la République, Paris, 1801, p. 280. «De

l'enthousiasme et de la sensibilité », note le critique.

42.Cité par H. Laurens, op. cit. note 8, p. 403.

43.Cf. P. Bret, L'Égypte au temps de l'expédition de Bonaparte (1798-1801), Paris, 1998, p. 220.

\section{RÉSUMÉS}

Les journaux de ses compagnons d'armes, les portraits peints ou écrits par les artistes qui le suivent, ne tarissent pas d'éloges sur Desaix. Le général est pourtant moins consensuel qu'il n'y paraît. Ses relations avec Bonaparte ou Kléber, égaux et concurrents, font preuve d'une franchise critique obstinée. Ses curiosités parfois naïves d'égyptologue se conjuguent avec sa rudesse de soldat en campagne, qui n'est pas forcément un soldat-citoyen. Sûr que sa "mission civilisatrice " justifie quelque réconfort de harem et, davantage encore, le règne de la peur (prises d'otages, peines d'une lourdeur exemplaire), Desaix s'attache aussi à connaître l'histoire, le mode de vie et la population des tribus, tentant un vaste fichier à vocation policière et diplomatique - il joue des affrontements ancestraux pour hâter le contrôle du territoire. Soucieux d'un amalgame des peuples au sein de l'armée, d'un développement économique et sanitaire aussi utile à la métropole qu'aux autochtones, il essaie d'appliquer sur le terrain les leçons de l'Institut du Caire, s'appuyant cependant davantage sur la minorité copte que sur les musulmans. Construisant une colonie, il construit aussi son image, à peine entachée par la capitulation d'al-'Arîsh, que Kleber lui impose, sciemment, de négocier, et qui le pousse immédiatement à chercher une réhabilitation auprès de Bonaparte.

\section{The Sultan Unveiled : Desaix in Egypt according to his Campaign Notebooks.}

The diaries of his fellow-soldiers, the portraits painted or written by the artists in attendance are redolent of praise for Desaix. The general, however, was less of a consensus figure than is apparent. His relations with Bonaparte or Kléber, his equals and competitors, were stubbornly critical and blunt. His somewhat naïve curiosity as an Egyptologist was allied to the roughness of a field officer who was not obviously a citizen-soldier. Certain that his "civilizing mission" justified the sparse comfort of a harem and even more a reign of fear (hostage-taking, exemplary harsh sentences), Desaix was also keen to investigate the history, way of life and make-up of the tribes, and as he built up a thick file for police and diplomatic use, he took advantage of ancestral rivalries to bring the land to heel. Intent on mixing indigenous groups within the army, on developing the local economy and sanitation for the good of the locals and the home country, he tried to implement in the field the lessons of the Cairo Institute, while relying more on the Coptic minority than the Moslems. In building a colony he also built his own image, barely tarnished by 
the capitulation of al'Arîsh, which Kléber deliberately forced him to negotiate and which immediately led him to seek rehabilitation from Bonaparte.

INDEX

Mots-clés : Desaix, campagne d'Égypte, soldat-citoyen, colonie, guerre de conquête

\section{AUTEUR}

PHILIPPE BOURDIN

Université Blaise-Pascal (Clermont-Ferrand II) 